International Journal of Pure and Applied Mathematics

Volume 116 No. 1 2017, 201-216

ISSN: 1311-8080 (printed version); ISSN: 1314-3395 (on-line version)

url: http://www.ijpam.eu

doi: 10.12732/ijpam.v116i1.22

ijpam.eu

\title{
A NOTE ON THE ALMOST SURE EXPONENTIAL STABILITY OF THE MILSTEIN METHOD FOR STOCHASTIC DELAY DIFFERENTIAL EQUATIONS WITH JUMPS
}

O. Farkhondeh Rouz ${ }^{1}$, D. Ahmadian ${ }^{2}$, A. Jodayree Akbarfam ${ }^{3}$, M. Milev ${ }^{4}$

${ }^{1,2,3}$ Faculty of Mathematical Sciences

University of Tabriz

Tabriz, IRAN

${ }^{4}$ Department of Mathematics and Physics

University of Food Technology-Plovdiv

Plovdiv, BULGARIA

\begin{abstract}
In this paper we consider the Milstein method to investigate the almost sure exponential stability for nonlinear stochastic delay differential equations (SDDEs) with jump diffusion, that is, processes that change only by jumps. The class of jump-diffusion SDDEs that admits explicit solutions is rather limited. In this regard, we employ the continuous and discrete semimartingale convergence theorem. It is shown that the Milstein method reproduces the almost sure exponential stability under an additional linear growth condition on drift and the function $L^{1} \mathrm{~g}$. In the numerical section we present the results by simulating the method on kind of equations.
\end{abstract}

AMS Subject Classification: $65 \mathrm{C} 20,60 \mathrm{H} 35,65 \mathrm{C} 30$

Key Words: Milstein method, almost sure exponential stability, jump-diffusion, nonlinear stochastic delay differential equations, semimartingale convergence theorem

\section{Introduction}

Jump diffusion is a stochastic process that involves jumps and diffusion. Mod-

Received: $\quad$ July 7,2017

Revised: $\quad$ August 21, 2017

Published: August 30, 2017

(c) 2017 Academic Publications, Ltd.

url: www.acadpubl.eu

$\S_{\text {Correspondence author }}$ 
els that incorporate jumps have become increasingly popular in finance and several areas of science and engineering. They have important applications in magnetic reconnection, coronal mass ejections, condensed matter physics, pattern theory and computational vision (see [27]). In particular, they are used in mathematical finance in order to simulate asset prices, interest rates and volatilities (see [2] and [7]). Jump models also are employed, appear in many other application areas and have proved successful at describing unexpected, abrupt changes of state [4]. Since only a limited class of jump-diffusion problems admit explicit solutions, it is imperative to develop numerical methods to solve these problems.

In special cases, stochastic delay differential equations (SDDEs) are a type of SDEs, which has been discussed in variety of sciences (see [9], [12] and [23]). Since most of these equations cannot be solved explicitly, numerical approximations become an important tool in studying the properties of these stochastic systems (see [1], [3], [10], [17], [20] and [26]). Since stability is one of the major concerns in systems analysis, the stability of numerical methods becomes also one of the main tools to examine the stability of the exact solution of the stochastic systems (see [18], [21] and [24]). Using the technique based on the continuous semimartingale convergence theorem, Mao [16] investigated the almost sure asymptotic stability of SDDEs.

Now efforts are being made to bring SDDEs with jumps up to a similar level. For SDDEs with jumps, the existing literature concerns mainly stability analysis of numerical methods. For example, Wang et al [11] constructed the semiimplicit Euler method for stochastic differential delay equation with jumps. Tan and Wang [5] investigated the mean-square stability of the explicit Euler method for linear SDDEs with jumps. Zhang et al [8] derived some criteria on pth moment stability and almost sure stability with general decay rates of stochastic differential delay equations with Poisson jumps and Markovian switching. Li et al [19] constructed compensated stochastic theta methods (CSTM) for stochastic differential delay equations with Poisson jumps, and got the mean square P-stability by some restriction on time stepsize.

Also, the stability of Milstein method has been applied to SDDEs. For example, [25] discusses the mean square stability of SDDEs with constant coefficients and [22] obtains also mean-square stability for SDDEs with integral part. Based on these papers, to the best of our knowledge, the alsmost sure exponential stability of Milstein method for SDDEs with jump has never been considered so far. In this paper, we derive a sufficient condition of the almost sure exponential stability of the Milstein method for SDDEs with jumps. These stability results show that the Milstein method reproduces the almost sure ex- 
ponential stability under an additional linear growth conditions on drift and the function $L^{1} g$.

The rest of the paper is organized as follows. Section 2 begins with notations and preliminaries, and establish the continuous semimartingale convergence theorem and discrete semimartingale convergence theorem. In Section 3 the Milstein method is introduced for SDDEs with jump. Section 4 examines the conditions under which the Milstein method can reproduce the almost sure exponential stability. Section 5 gives the numerical experiments to confirm the theoretical results.

\section{Preliminaries and notations}

Throughout this paper, unless otherwise specified, we use the following notations. Let $|\cdot|$ denotes both the Euclidean norm in $\mathbb{R}^{n}$ and the trace (or Frobenius) norm in $\mathbb{R}^{n \times d}$. The inner product of $x, y \in \mathbb{R}^{n}$ is denoted by $\langle x, y\rangle$ or $x^{T} y$. If $A$ is a vector or matrix, its transpose is denoted by $A^{T}$. If $\mathrm{A}$ is a matrix, its trace norm is denoted by $|A|=\sqrt{\operatorname{trace}\left(A^{T} A\right)} . a \vee b$ represents $\max \{a, b\}$ and $a \wedge b$ denotes $\min \{a, b\}$. Let $(\Omega, \mathcal{F}, \mathbf{P})$ be a complete probability space with a filtration $\left\{\mathcal{F}_{t}\right\}_{t \geq 0}$, which is right continuous and satisfies that each $\mathcal{F}_{0}$ contains all $\mathbf{P}$-null sets. Denote by $C\left([-\tau, 0] ; \mathbb{R}^{n}\right)$ the family of continuous functions from $[-\tau, 0]$ to $\mathbb{R}^{n}$ equipped with the norm $\|\psi\|:=\sup _{-\tau \leq t \leq 0}|\psi(t)|$. Let $W(t)$ be a $d$-dimensional standard Brownian motion defined on this probability space and $N(t)$ be a scalar Poisson process with parameter $\lambda>0$ and independent of the Brownian motion. Let $f: \mathbb{R}^{+} \times \mathbb{R}^{n} \times \mathbb{R}^{n} \mapsto \mathbb{R}^{n}, g: \mathbb{R}^{+} \times \mathbb{R}^{n} \times \mathbb{R}^{n} \mapsto \mathbb{R}^{n \times d}$ and $h: \mathbb{R}^{+} \times \mathbb{R}^{n} \times \mathbb{R}^{n} \mapsto \mathbb{R}^{n}$ are continuous functions.

Let us consider the $n$-dimensional SDDE with jump-diffusion of the form

$$
\begin{aligned}
d x(t) & =f(t, x(t), x(t-\tau)) d t+g(t, x(t), x(t-\tau)) d W(t) \\
& +h\left(t, x\left(t^{-}\right), x\left(t^{-}-\tau\right)\right) d N(t), \quad t>0
\end{aligned}
$$

with initial data $x(t)=\psi(t) \in C\left([-\tau, 0] ; \mathbb{R}^{n}\right)$, where the delay $\tau>0$ is a fixed constant, $x\left(t^{-}\right)$denotes $\lim _{s \rightarrow t^{-}} x(s)$. As a standing hypothesis, we assume that the functions $f, g$ and $h$ satisfy the local Lipschitz condition:

Assumption 1. The functions $f, g$ and $h$ satisfy the local Lipschitz condition, that is, for each $j>0$ there exists a positive constant $C_{j}$ such that for all $t \geq 0$ and any $x, y, \bar{x}, \bar{y} \in \mathbb{R}^{n}$ with $|x| \vee|y| \vee|\bar{x}| \vee|\bar{y}| \leq j$,

$$
|f(t, x, y)-f(t, \bar{x}, \bar{y})|^{2} \vee|g(t, x, y)-g(t, \bar{x}, \bar{y})|^{2} \vee|h(t, x, y)-h(t, \bar{x}, \bar{y})|^{2} \leq C_{j}\left(|x-\bar{x}|^{2}+|y-\bar{y}|^{2}\right)
$$


To be precise, we give the definition on the almost sure exponential stability of the SDDEs with jumps.

Definition 1. The solution $x(t)$ to Equation (1) is said to be almost surely exponentially stable if there exists a constant $\eta>0$ such that

$$
\limsup _{t \rightarrow \infty} \frac{1}{t} \log |x(t)| \leq-\eta \quad \text { a.s. }
$$

for any initial data $\psi(t) \in C\left([-\tau, 0] ; \mathbb{R}^{n}\right)$.

In the theory of stochastic processes in discrete time, a part of the mathematical theory of probability, the Doob decomposition theorem, gives a unique decomposition of every adapted and integrable stochastic process as the sum of a martingale and a predictable process starting at zero. The following lemmas will play important roles in this paper.

Lemma 2. (Continuous Semimartingale Convergence Theorem, see [28] and [14]). Let $\{A(t)\},\{U(t)\}$ be two $\mathcal{F}_{t}$-adapted increasing processes on $t \geq 0$ with $A(0)=U(0)=0$ a.s. Let $M(t)$ be a real-valued local martingale with $M_{0}=0$ a.s. Let $\zeta$ be a nonnegative $\mathcal{F}_{0}$-measurable random variable. Assume that $\{X(t)\}$ is nonnegative and

$$
X(t)=\zeta+A(t)-U(t)+M(t) \text { for } t \geq 0 .
$$

If $\lim _{t \rightarrow \infty} A(t)<\infty$ a.s., then for almost all $W \in \Omega$,

$$
\lim _{t \rightarrow \infty} X(t)<\infty \text { and } \lim _{t \rightarrow \infty} U(t)<\infty,
$$

that is, both $X(t)$ and $U(t)$ converge to finite random variables.

Lemma 3. (Discrete Semimartingale Convergence Theorem, see [6] and [29]). Let $\left\{A_{i}\right\},\left\{U_{i}\right\}$ be two sequences of nonnegative random variables such that both $A_{i}$ and $U_{i}$ are $\mathcal{F}_{i}$-measurable for $i=1,2, \cdots$, and $A_{0}=U_{0}=0$ a.s. Let $M_{i}$ be a real-valued local martingale with $M_{0}=0$ a.s. Let $\zeta$ be a nonnegative $\mathcal{F}_{0}$-measurable random variable. Assume that $\left\{X_{i}\right\}$ is a nonnegative semimartingale with the Doob-Mayer decomposition

$$
X_{i}=\zeta+A_{i}-U_{i}+M_{i}
$$

If $\lim _{i \rightarrow \infty} A_{i}<\infty$ a.s., then for almost all $W \in \Omega$,

$$
\lim _{i \rightarrow \infty} X_{i}<\infty \text { and } \lim _{i \rightarrow \infty} U_{i}<\infty,
$$

that is, both $X_{i}$ and $U_{i}$ converge to finite random variables. 
We are now ready to formulate our first result, which provide us with a criterion on the almost sure exponential stability of the exact solution to Equation (1).

Theorem 4. Let Assumption 1 holds. Assume that there exist positive constants $\alpha_{i}, \beta_{i}, \gamma_{i}, i=1,2$ such that for any $x, y \in \mathbb{R}^{n}$ and $t \geq 0$

$$
\begin{aligned}
\langle x, f(t, x, 0)\rangle & \leq-\alpha_{1}|x|^{2}, \\
|f(t, x, y)-f(t, x, 0)| & \leq \alpha_{2}|y|, \\
|g(t, x, y)| & \leq \beta_{1}|x|+\beta_{2}|y|, \\
|h(t, x, y)| & \leq \gamma_{1}|x|+\gamma_{2}|y| .
\end{aligned}
$$

If

$$
2 \alpha_{1}>2 \alpha_{2}+\left(\beta_{1}+\beta_{2}\right)^{2}+\lambda\left(2\left(\gamma_{1}+\gamma_{2}\right)+\left(\gamma_{1}+\gamma_{2}\right)^{2}\right)
$$

then in [19] Li et al, show that for any given initial data $\psi(t)$, there exists a unique global solution $x(t)$ to Equation (1) and the solution has the property that

$$
\limsup _{t \rightarrow \infty} \frac{1}{t} \log |x(t)| \leq-\frac{\gamma}{2} \quad \text { a.s. }
$$

where $\gamma>0$ is the unique positive root of

$$
\begin{aligned}
\gamma+\left(\beta_{1}^{2}+\beta_{1} \beta_{2}\right. & \left.+\lambda\left(\gamma_{1}^{2}+2 \gamma_{1}+\gamma_{1} \gamma_{2}+\gamma_{2}\right)-2 \alpha_{1}+\alpha_{2}\right) \\
& +\left(\beta_{2}^{2}+\beta_{1} \beta_{2}+\lambda\left(\gamma_{2}^{2}+\gamma_{1} \gamma_{2}+\gamma_{2}\right)+\alpha_{2}\right) e^{\gamma \tau}=0
\end{aligned}
$$

\section{The Milstein method on SDDEs with jumps}

Now we introduce the Milstein approximation $\left\{y_{k}\right\}_{k \geq 0}$ as follows:

$$
\begin{aligned}
y_{k+1} & =y_{k}+f\left(t_{k}, y_{k}, y_{k-m}\right) \Delta t+g\left(t_{k}, y_{k}, y_{k-m}\right) \Delta W_{k} \\
& +\frac{1}{2} L^{1} g\left(t_{k}, y_{k}, y_{k-m}\right)\left(\left|\Delta W_{k}\right|^{2}-\Delta t\right)+h\left(t_{k}, y_{k}, y_{k-m}\right) \Delta N_{k}, \quad k=0,1,2, \cdots,
\end{aligned}
$$

where $L^{1}=g(x) \frac{\partial}{\partial x}$, and stepsize $\Delta t=\frac{\tau}{m}$ for a integer $m, y_{k}=\psi(k \Delta t)$ for $k=-m, \ldots, 0, y_{0}=\psi(0)$, whose value of $\Delta t$ may change with each appearance. The Brownian increments is defined as $\Delta W_{k}:=W\left(t_{k+1}\right)-W\left(t_{k}\right)$ and $\Delta N_{k}:=N\left(t_{k+1}\right)-N\left(t_{k}\right)$ represent the Poisson process, respectively. 
Assumption 2. Assume that the function $L^{1} g$ satisfy the local Lipschitz condition, that is, there exists a constant $K_{1}$ such that for any $x, y, \bar{x}, \bar{y} \in \mathbb{R}^{n}$ and $t>0$,

$$
\left|L^{1} g(t, x, y)-L^{1} g(t, \bar{x}, \bar{y})\right|^{2} \leq K_{1}\left(|x-\bar{x}|^{2}+|y-\bar{y}|^{2}\right) .
$$

For the purpose of stability, we assume that $f(t, 0,0)=g(t, 0,0)=h(t, 0,0)=$ 0 . This shows that Equation (1) admits a trivial solution, then inequality (16) becomes

$$
\left|L^{1} g(t, x, y)\right|^{2} \leq K_{1}\left(|x|^{2}+|y|^{2}\right) .
$$

To be precise, the definition on the almost sure exponential stability of the numerical solutions is given as follows:

Definition 5. The numerical solution $\left\{y_{k}\right\}_{k \geq 0}$ to Equation (15) is said to be almost surely exponentially stable, if there exists a constant $\bar{\eta}>0$ such that

$$
\limsup _{k \rightarrow \infty} \frac{1}{k \Delta t} \log \left|y_{k}\right| \leq-\bar{\eta} \quad \text { a.s. }
$$

for any initial data $\psi(k \Delta t)$ for $k=-m,-m+1, \ldots, 0$.

\section{Stability analysis of the Milstein method on SDDEs with jumps}

In this section, we assume that $f, g$ and $h$ satisfy the local lipschitz condition. Now we investigate the almost sure stability analysis of Milstein approximation $\left\{y_{k}\right\}_{k \geq 0}$ with jumps.

Theorem 6. Let conditions (8)-(12) hold and the function $L^{1} g$ satisfies the linear growth condition (17). If there exists a positive constant $K_{2}$ such that $f$ satisfies the linear growth condition

$$
|f(t, x, y)|^{2} \leq K_{2}\left(|x|^{2}+|y|^{2}\right), \quad \text { for all }(x, y) \in \mathbb{R}^{n} \times \mathbb{R}^{n},
$$

then there is a stepsize bound $\Delta t^{*}>0$, such that for any $\Delta t<\Delta t^{*}$, the Milstein approximation $\left\{y_{k}\right\}_{k \geq 0}$ has the property

$$
\limsup _{k \rightarrow \infty} \frac{1}{k \Delta t} \log \left|y_{k}\right| \leq-\frac{\bar{\eta}}{2}+\varepsilon \quad \text { a.s. }
$$

where $\bar{\eta}>0$ is the unique root of the equation 


$$
\begin{aligned}
\bar{\eta}+\left(\beta_{1}^{2}+\beta_{1} \beta_{2}\right. & \left.+\lambda\left(\gamma_{1}^{2}+2 \gamma_{1}+\gamma_{1} \gamma_{2}+\gamma_{2}\right)-2 \alpha_{1}+\alpha_{2}\right) \\
& +\left(\beta_{2}^{2}+\beta_{1} \beta_{2}+\lambda\left(\gamma_{2}^{2}+\gamma_{1} \gamma_{2}+\gamma_{2}\right)+\alpha_{2}\right) e^{\bar{\eta} \tau}=0
\end{aligned}
$$

and $\varepsilon \in\left(0, \frac{\bar{\eta}}{2}\right)$ is arbitrary.

Proof. By using the martingale property of the compensated Poisson process

$$
\tilde{N}(t)=N(t)-\lambda t
$$

we rewrite (15) in the form of

$$
\begin{aligned}
y_{k+1} & =y_{k}+\left(f\left(t_{k}, y_{k}, y_{k-m}\right)+\lambda h\left(t_{k}, y_{k}, y_{k-m}\right)\right) \Delta t+g\left(t_{k}, y_{k}, y_{k-m}\right) \Delta W_{k} \\
& +\frac{1}{2} L^{1} g\left(t_{k}, y_{k}, y_{k-m}\right)\left(\left|\Delta W_{k}\right|^{2}-\Delta t\right)+h\left(t_{k}, y_{k}, y_{k-m}\right) \Delta \tilde{N}_{k}, \quad k=0,1,2, \cdots,
\end{aligned}
$$

where $\Delta \tilde{N}_{k}=\tilde{N}\left(t_{k+1}\right)-\tilde{N}\left(t_{k}\right)$.

It is easy to deduce from (23) that

$$
\begin{aligned}
\left|y_{k+1}\right|^{2} & =\left|y_{k}\right|^{2}+\left|f\left(t_{k}, y_{k}, y_{k-m}\right)+\lambda h\left(t_{k}, y_{k}, y_{k-m}\right)\right|^{2}(\Delta t)^{2}+\left|g\left(t_{k}, y_{k}, y_{k-m}\right)\right|^{2}\left|\Delta W_{k}\right|^{2} \\
& +\frac{1}{4}\left|L^{1} g\left(t_{k}, y_{k}, y_{k-m}\right)\left(\left|\Delta W_{k}\right|^{2}-\Delta t\right)\right|^{2}+\left|h\left(t_{k}, y_{k}, y_{k-m}\right)\right|^{2}\left|\Delta \tilde{N}_{k}\right|^{2} \\
& +2\left\langle y_{k}, f\left(t_{k}, y_{k}, y_{k-m}\right)\right\rangle \Delta t+2 \lambda \Delta t\left\langle y_{k}, h\left(t_{k}, y_{k}, y_{k-m}\right)\right\rangle+2\left\langle y_{k}, g\left(t_{k}, y_{k}, y_{k-m}\right)\right\rangle \Delta W_{k} \\
& +\left\langle y_{k}, L^{1} g\left(t_{k}, y_{k}, y_{k-m}\right)\left(\left|\Delta W_{k}\right|^{2}-\Delta t\right)\right\rangle+2\left\langle y_{k}, h\left(t_{k}, y_{k}, y_{k-m}\right)\right\rangle \Delta \tilde{N}_{k} \\
& +2 \Delta t\left\langle f\left(t_{k}, y_{k}, y_{k-m}\right)+\lambda h\left(t_{k}, y_{k}, y_{k-m}\right), g\left(t_{k}, y_{k}, y_{k-m}\right) \Delta W_{k}\right\rangle \\
& +2 \Delta t\left\langle f\left(t_{k}, y_{k}, y_{k-m}\right)+\lambda h\left(t_{k}, y_{k}, y_{k-m}\right), L^{1} g\left(t_{k}, y_{k}, y_{k-m}\right)\left(\left|\Delta W_{k}\right|^{2}-\Delta t\right)\right\rangle \\
& +2 \Delta t\left\langle f\left(t_{k}, y_{k}, y_{k-m}\right)+\lambda h\left(t_{k}, y_{k}, y_{k-m}\right), h\left(t_{k}, y_{k}, y_{k-m}\right) \Delta \tilde{N}_{k}\right\rangle \\
& +\left\langle g\left(t_{k}, y_{k}, y_{k-m}\right) \Delta W_{k}, L^{1} g\left(t_{k}, y_{k}, y_{k-m}\right)\left(\left|\Delta W_{k}\right|^{2}-\Delta t\right)\right\rangle \\
& +\left\langle L^{1} g\left(t_{k}, y_{k}, y_{k-m}\right)\left(\left|\Delta W_{k}\right|^{2}-\Delta t\right), h\left(t_{k}, y_{k}, y_{k-m}\right) \Delta \tilde{N}_{k}\right\rangle \\
& +2\left\langle g\left(t_{k}, y_{k}, y_{k-m}\right) \Delta W_{k}, h\left(t_{k}, y_{k}, y_{k-m}\right) \Delta \tilde{N}_{k}\right\rangle .
\end{aligned}
$$

By using the elementary inequality $(a+b)^{2} \leq 2 a^{2}+2 b^{2}$ and condition (11), we have

$$
\begin{gathered}
\left|f\left(t_{k}, y_{k}, y_{k-m}\right)+\lambda h\left(t_{k}, y_{k}, y_{k-m}\right)\right|^{2}(\Delta t)^{2} \leq 2 K_{2}(\Delta t)^{2}\left(\left|y_{k}\right|^{2}+\left|y_{k-m}\right|^{2}\right) \\
+2 \lambda^{2}(\Delta t)^{2}\left[\left(\gamma_{1}^{2}+\gamma_{1} \gamma_{2}\right)\left|y_{k}\right|^{2}+\left(\gamma_{2}^{2}+\gamma_{1} \gamma_{2}\right)\left|y_{k-m}\right|^{2}\right]
\end{gathered}
$$

and by conditions (8)-(10), we obtain $2\left\langle y_{k}, f\left(t_{k}, y_{k}, y_{k-m}\right)\right\rangle=2\left\langle y_{k}, f\left(t_{k}, y_{k}, 0\right)\right\rangle+2\left\langle y_{k}, f\left(t_{k}, y_{k}, y_{k-m}\right)-f\left(t_{k}, y_{k}, 0\right)\right\rangle$ 


$$
\begin{gathered}
\leq-2 \alpha_{1}\left|y_{k}\right|^{2}+2 \alpha_{2}\left|y_{k}\right|\left|y_{k-m}\right| \\
\leq\left(-2 \alpha_{1}+\alpha_{2}\right)\left|y_{k}\right|^{2}+\alpha_{2}\left|y_{k-m}\right|^{2} . \\
\left|g\left(t_{k}, y_{k}, y_{k-m}\right)\right|^{2}\left|\Delta W_{k}\right|^{2} \leq \Delta t\left[\left(\beta_{1}^{2}+\beta_{1} \beta_{2}\right)\left|y_{k}\right|^{2}+\left(\beta_{2}^{2}+\beta_{1} \beta_{2}\right)\left|y_{k-m}\right|^{2}\right] \\
+\left|g\left(t_{k}, y_{k}, y_{k-m}\right)\right|^{2}\left(\left|\Delta W_{k}\right|^{2}-\Delta t\right) .
\end{gathered}
$$

Also by using the linear growth condition (17), we have

$$
\begin{aligned}
\frac{1}{4}\left|L^{1} g\left(t_{k}, y_{k}, y_{k-m}\right)\left(\left|\Delta W_{k}\right|^{2}-\Delta t\right)\right|^{2} & =\frac{1}{4}\left|L^{1} g\left(t_{k}, y_{k}, y_{k-m}\right)\right|^{2}\left[\left(\left|\Delta W_{k}\right|^{2}-\Delta t\right)^{2}-2(\Delta t)^{2}\right] \\
& +\frac{1}{2}\left|L^{1} g\left(t_{k}, y_{k}, y_{k-m}\right)\right|^{2}(\Delta t)^{2} \\
& \leq \frac{1}{4}\left|L^{1} g\left(t_{k}, y_{k}, y_{k-m}\right)\right|^{2}\left[\left(\left|\Delta W_{k}\right|^{2}-\Delta t\right)^{2}-2(\Delta t)^{2}\right] \\
& +\frac{1}{2} K_{1}\left(\left|y_{k}\right|^{2}+\left|y_{k-m}\right|^{2}\right)(\Delta t)^{2} .
\end{aligned}
$$

Now substituting (25), (26), (27) and (28) into (24), we obtain

$$
\begin{aligned}
\left|y_{k+1}\right|^{2} \leq & \left|y_{k}\right|^{2}+2 K_{2}(\Delta t)^{2}\left(\left|y_{k}\right|^{2}+\left|y_{k-m}\right|^{2}\right)+2 \lambda^{2}(\Delta t)^{2}\left[\left(\gamma_{1}^{2}+\gamma_{1} \gamma_{2}\right)\left|y_{k}\right|^{2}\right. \\
& \left.+\left(\gamma_{2}^{2}+\gamma_{1} \gamma_{2}\right)\left|y_{k-m}\right|^{2}\right]+\Delta t\left[\left(\beta_{1}^{2}+\beta_{1} \beta_{2}\right)\left|y_{k}\right|^{2}+\left(\beta_{2}^{2}+\beta_{1} \beta_{2}\right)\left|y_{k-m}\right|^{2}\right] \\
& +\left|g\left(t_{k}, y_{k}, y_{k-m}\right)\right|^{2}\left(\left|\Delta W_{k}\right|^{2}-\Delta t\right)+\frac{1}{2} K_{1}\left(\left|y_{k}\right|^{2}+\left|y_{k-m}\right|^{2}\right)(\Delta t)^{2} \\
& +\frac{1}{4}\left|L^{1} g\left(t_{k}, y_{k}, y_{k-m}\right)\right|^{2}\left[\left(\left|\Delta W_{k}\right|^{2}-\Delta t\right)^{2}-2(\Delta t)^{2}\right]+\lambda \Delta t\left[\left(\gamma_{1}^{2}+\gamma_{1} \gamma_{2}\right)\left|y_{k}\right|^{2}\right. \\
& \left.+\left(\gamma_{2}^{2}+\gamma_{1} \gamma_{2}\right)\left|y_{k-m}\right|^{2}\right]+\left|h\left(t_{k}, y_{k}, y_{k-m}\right)\right|^{2}\left(\Delta \tilde{N}_{k}^{2}-\lambda \Delta t\right)+\Delta t\left[\left(-2 \alpha_{1}+\alpha_{2}\right)\left|y_{k}\right|^{2}\right. \\
& \left.+\alpha_{2}\left|y_{k-m}\right|^{2}\right]+\lambda \Delta t\left[\left(2 \gamma_{1}+\gamma_{2}\right)\left|y_{k}\right|^{2}+\gamma_{2}\left|y_{k-m}\right|^{2}\right]+2\left\langle y_{k}, g\left(t_{k}, y_{k}, y_{k-m}\right) \Delta W_{k}\right\rangle \\
& +2\left\langle y_{k}, L^{1} g\left(t_{k}, y_{k}, y_{k-m}\right)\left(\left|\Delta W_{k}\right|^{2}-\Delta t\right)\right\rangle+2\left\langle y_{k}, h\left(t_{k}, y_{k}, y_{k-m}\right) \Delta \tilde{N}_{k}\right\rangle \\
& +2 \Delta t\left\langle f\left(t_{k}, y_{k}, y_{k-m}\right)+\lambda h\left(t_{k}, y_{k}, y_{k-m}\right), g\left(t_{k}, y_{k}, y_{k-m}\right) \Delta W_{k}\right\rangle \\
& +\Delta t\left\langle f\left(t_{k}, y_{k}, y_{k-m}\right)+\lambda h\left(t_{k}, y_{k}, y_{k-m}\right), L^{1} g\left(t_{k}, y_{k}, y_{k-m}\right)\left(\left|\Delta W_{k}\right|^{2}-\Delta t\right)\right\rangle \\
& +2 \Delta t\left\langle f\left(t_{k}, y_{k}, y_{k-m}\right)+\lambda h\left(t_{k}, y_{k}, y_{k-m}\right), h\left(t_{k}, y_{k}, y_{k-m}\right) \Delta \tilde{N}_{k}\right\rangle \\
& +\left\langle g\left(t_{k}, y_{k}, y_{k-m}\right) \Delta W_{k}, L^{1} g\left(t_{k}, y_{k}, y_{k-m}\right)\left(\left|\Delta W_{k}\right|^{2}-\Delta t\right)\right\rangle \\
& +\left\langle L^{1} g\left(t_{k}, y_{k}, y_{k-m}\right)\left(\left|\Delta W_{k}\right|^{2}-\Delta t\right), h\left(t_{k}, y_{k}, y_{k-m}\right) \Delta \tilde{N}_{k}\right\rangle \\
& +2\left\langle g\left(t_{k}, y_{k}, y_{k-m}\right) \Delta W_{k}, h\left(t_{k}, y_{k}, y_{k-m}\right) \Delta \tilde{N}_{k}\right\rangle \\
& =\mu\left|y_{k}\right|^{2}+\sigma\left|y_{k-m}\right|^{2}+m_{k}^{\Delta},
\end{aligned}
$$

where

$$
\mu=1+\left[2 K_{2} \Delta t+2 \lambda^{2} \Delta t\left(\gamma_{1}^{2}+\gamma_{1} \gamma_{2}\right)+\beta_{1}^{2}+\beta_{1} \beta_{2}+\frac{1}{2} K_{1} \Delta t\right.
$$




$$
\begin{aligned}
+ & \left.\lambda\left(\gamma_{1}^{2}+2 \gamma_{1}+\gamma_{1} \gamma_{2}+\gamma_{2}\right)-2 \alpha_{1}+\alpha_{2}\right] \Delta t \\
\sigma= & {\left[2 K_{2} \Delta t+2 \lambda^{2} \Delta t\left(\gamma_{1}^{2}+\gamma_{1} \gamma_{2}\right)+\beta_{1}^{2}+\beta_{1} \beta_{2}+\frac{1}{2} K_{1} \Delta t\right.} \\
& \left.+\lambda\left(\gamma_{1}^{2}+\gamma_{1} \gamma_{2}+\gamma_{2}\right)+\alpha_{2}\right] \Delta t
\end{aligned}
$$

and

$$
\begin{aligned}
m_{k}^{\Delta} & =\left|g\left(t_{k}, y_{k}, y_{k-m}\right)\right|^{2}\left(\left|\Delta W_{k}\right|^{2}-\Delta t\right)+\frac{1}{4}\left|L^{1} g\left(t_{k}, y_{k}, y_{k-m}\right)\right|^{2}\left[\left(\left|\Delta W_{k}\right|^{2}-\Delta t\right)^{2}\right. \\
& \left.-2(\Delta t)^{2}\right]+\left|h\left(t_{k}, y_{k}, y_{k-m}\right)\right|^{2}\left(\Delta \tilde{N}_{k}^{2}-\lambda \Delta t\right)+2\left\langle y_{k}, g\left(t_{k}, y_{k}, y_{k-m}\right) \Delta W_{k}\right\rangle \\
& +\left\langle y_{k}, L^{1} g\left(t_{k}, y_{k}, y_{k-m}\right)\left(\left|\Delta W_{k}\right|^{2}-\Delta t\right)\right\rangle+2\left\langle y_{k}, h\left(t_{k}, y_{k}, y_{k-m}\right) \Delta \tilde{N}_{k}\right\rangle \\
& +2 \Delta t\left\langle f\left(t_{k}, y_{k}, y_{k-m}\right)+\lambda h\left(t_{k}, y_{k}, y_{k-m}\right), g\left(t_{k}, y_{k}, y_{k-m}\right) \Delta W_{k}\right\rangle \\
& +\Delta t\left\langle f\left(t_{k}, y_{k}, y_{k-m}\right)+\lambda h\left(t_{k}, y_{k}, y_{k-m}\right), L^{1} g\left(t_{k}, y_{k}, y_{k-m}\right)\left(\left|\Delta W_{k}\right|^{2}-\Delta t\right)\right\rangle \\
& +2 \Delta t\left\langle f\left(t_{k}, y_{k}, y_{k-m}\right)+\lambda h\left(t_{k}, y_{k}, y_{k-m}\right), h\left(t_{k}, y_{k}, y_{k-m}\right) \Delta \tilde{N}_{k}\right\rangle \\
& +\left\langle g\left(t_{k}, y_{k}, y_{k-m}\right) \Delta W_{k}, L^{1} g\left(t_{k}, y_{k}, y_{k-m}\right)\left(\left|\Delta W_{k}\right|^{2}-\Delta t\right)\right\rangle \\
& +\left\langle L^{1} g\left(t_{k}, y_{k}, y_{k-m}\right)\left(\left|\Delta W_{k}\right|^{2}-\Delta t\right), h\left(t_{k}, y_{k}, y_{k-m}\right) \Delta \tilde{N}_{k}\right\rangle \\
& +2\left\langle g\left(t_{k}, y_{k}, y_{k-m}\right) \Delta W_{k}, h\left(t_{k}, y_{k}, y_{k-m}\right) \Delta \tilde{N}_{k}\right\rangle .
\end{aligned}
$$

For any positive number $R \geq 1$, we have

$$
\begin{aligned}
R^{(k+1) \Delta t}\left|y_{k+1}\right|^{2}-R^{k \Delta t}\left|y_{k}\right|^{2} & \leq\left(\mu-R^{-\Delta t}\right) R^{(k+1) \Delta t}\left|y_{k}\right|^{2} \\
& +\sigma R^{(k+1) \Delta t}\left|y_{k-m}\right|^{2}+R^{(k+1) \Delta t} m_{k}^{\Delta} .
\end{aligned}
$$

Summing from $j=0$ to $j=k-1$, which implies

$$
\begin{aligned}
R^{k \Delta t}\left|y_{k}\right|^{2} & \leq\left|y_{0}\right|^{2}+\left(\mu-R^{-\Delta t}\right) \sum_{j=0}^{k-1} R^{(j+1) \Delta t}\left|y_{j}\right|^{2} \\
& +\sigma \sum_{j=0}^{k-1} R^{(j+1) \Delta t}\left|y_{j-m}\right|^{2}+M_{k}(R)
\end{aligned}
$$

where $M_{k}(R)=\sum_{j=0}^{k-1} R^{(j+1) \Delta t} m_{j}^{\Delta}$. Now, we are in the position to show that $M_{k}(R)$ is a martingale. By [26] it is easy to see that

$$
\sum_{j=0}^{k-1} R^{(j+1) \Delta t}\left|g\left(t_{j}, y_{j}, y_{j-m}\right)\right|^{2}\left(\left|\Delta W_{j}\right|^{2}-\Delta t\right)+2\left\langle y_{j}, g\left(t_{j}, y_{j}, y_{j-m}\right) \Delta W_{j}\right\rangle+2\left\langle f\left(t_{j}, y_{j}, y_{j-m}\right)\right.
$$




$$
\left.+\lambda h\left(t_{j}, y_{j}, y_{j-m}\right), g\left(t_{j}, y_{j}, y_{j-m}\right) \Delta W_{j}\right\rangle \Delta t
$$

is a martingale. Also

$$
\begin{gathered}
\sum_{j=0}^{k-1} R^{(j+1) \Delta t} \frac{1}{4}\left|L^{1} g\left(t_{j}, y_{j}, y_{j-m}\right)\right|^{2}\left[\left(\left|\Delta W_{j}\right|^{2}-\Delta t\right)^{2}-2(\Delta t)^{2}\right] \\
+\left\langle y_{j}, L^{1} g\left(t_{j}, y_{j}, y_{j-m}\right)\left(\left|\Delta W_{j}\right|^{2}-\Delta t\right)\right\rangle+\left\langle f\left(t_{j}, y_{j}, y_{j-m}\right)\right. \\
\left.+\lambda h\left(t_{j}, y_{j}, y_{j-m}\right), L^{1} g\left(t_{j}, y_{j}, y_{j-m}\right)\left(\left|\Delta W_{j}\right|^{2}-\Delta t\right)\right\rangle \Delta t
\end{gathered}
$$

is a martingale.

Let $\widehat{m}_{k}^{\Delta}=\sum_{j=0}^{k-1} R^{(j+1) \Delta t}\left|h\left(t_{j}, y_{j}, y_{j-m}\right)\right|^{2}\left(\Delta \tilde{N}_{j}^{2}-\lambda \Delta t\right)$. Noting that $\mathbb{E}\left[\left(\Delta \tilde{N}_{j}^{2}-\right.\right.$ $\left.\lambda \Delta t) \mid \mathcal{F}_{j \Delta t}\right]=0$ and $y_{j}$ is $\mathcal{F}_{j \Delta t}$-measurable, then we have

$$
\begin{aligned}
\mathbb{E}\left[\widehat{m}_{k}^{\Delta} \mid \mathcal{F}_{(k-1) \Delta t}\right] & =\widehat{m}_{k-1}^{\Delta}+\mathbb{E}\left[R^{k \Delta t}\left|h\left(t_{k-1}, y_{k-1}, y_{k-m-1}\right)\right|^{2}\left(\Delta \tilde{N}_{k-1}^{2}-\lambda \Delta t\right) \mid \mathcal{F}_{(k-1) \Delta t}\right] \\
& =\widehat{m}_{k-1}^{\Delta}+R^{k \Delta t}\left|h\left(t_{k-1}, y_{k-1}, y_{k-m-1}\right)\right|^{2} \mathbb{E}\left[\left(\Delta \tilde{N}_{k-1}^{2}-\lambda \Delta t\right) \mid \mathcal{F}_{(k-1) \Delta t}\right] \\
& =\widehat{m}_{k-1}^{\Delta},
\end{aligned}
$$

which implies that $\widehat{m}_{k}^{\Delta}$ is a martingale. Similarly, $2 \sum_{j=0}^{k-1} R^{(j+1) \Delta t}\left\langle y_{j}, h\left(t_{j}, y_{j}, y_{j-m}\right)\right\rangle \Delta \tilde{N}_{j}$ and $2 \Delta t \sum_{j=0}^{k-1} R^{(j+1) \Delta t}\left\langle f\left(t_{j}, y_{j}, y_{j-m}\right)+\lambda h\left(t_{j}, y_{j}, y_{j-m}\right), h\left(t_{j}, y_{j}, y_{j-m}\right)\right\rangle \Delta \tilde{N}_{j}$ are martingales. By the independence of $W(t)$ and $\tilde{N}(t)$, similarly both of the following terms

$$
\sum_{j=0}^{k-1} R^{(j+1) \Delta t}\left\langle L^{1} g\left(t_{j}, y_{j}, y_{j-m}\right)\left(\left|\Delta W_{j}\right|^{2}-\Delta t\right), h\left(t_{j}, y_{j}, y_{j-m}\right) \Delta \tilde{N}_{j}\right\rangle
$$

and

$$
2 \sum_{j=0}^{k-1} R^{(j+1) \Delta t}\left\langle g\left(t_{j}, y_{j}, y_{j-m}\right) \Delta W_{j}, h\left(t_{j}, y_{j}, y_{j-m}\right) \Delta \tilde{N}_{j}\right\rangle,
$$

are martingales. This implies that $M_{k}(R)$ is a martingale with $M_{0}(R)=0$.

We know that

$$
\begin{aligned}
\sum_{j=0}^{k-1} R^{(j+1) \Delta t}\left|y_{j-m}\right|^{2} & =\sum_{j=-m}^{-1} R^{(j+m+1) \Delta t}\left|y_{j}\right|^{2}+\sum_{j=0}^{k-1} R^{(j+m+1) \Delta t}\left|y_{j}\right|^{2} \\
& -\sum_{j=k-m}^{k-1} R^{(j+m+1) \Delta t}\left|y_{j}\right|^{2}
\end{aligned}
$$


Substituting inequality (36) into (34) yields

$$
\begin{aligned}
R^{k \Delta t}\left|y_{k}\right|^{2}+\sigma \sum_{j=k-m}^{k-1} R^{(j+m+1) \Delta t}\left|y_{j}\right|^{2} & \leq\left|y_{0}\right|^{2}+h_{\Delta}(R) \sum_{j=0}^{k-1} R^{(j+1) \Delta t}\left|y_{j}\right|^{2} \\
& +\sigma \sum_{j=-m}^{-1} R^{(j+m+1) \Delta t}\left|y_{j}\right|^{2}+M_{k}(R),
\end{aligned}
$$

where

$$
\begin{aligned}
h_{\Delta}(R) & =R^{\Delta t}\left(\mu-R^{-\Delta t}+\sigma R^{m \Delta t}\right) \\
& =\left[1+\left(2 K_{2} \Delta t+2 \lambda^{2} \Delta t\left(\gamma_{1}^{2}+\gamma_{1} \gamma_{2}\right)+\beta_{1}^{2}+\beta_{1} \beta_{2}+\frac{1}{2} K_{1} \Delta t\right.\right. \\
& \left.\left.+\lambda\left(\gamma_{1}^{2}+2 \gamma_{1}+\gamma_{1} \gamma_{2}+\gamma_{2}\right)-2 \alpha_{1}+\alpha_{2}\right) \Delta t\right] R^{\Delta t} \\
& +\left[2 K_{2} \Delta t+2 \lambda^{2} \Delta t\left(\gamma_{2}^{2}+\gamma_{1} \gamma_{2}\right)+\beta_{2}^{2}+\beta_{1} \beta_{2}+\frac{1}{2} K_{1} \Delta t\right. \\
& \left.+\lambda\left(\gamma_{2}^{2}+\gamma_{1} \gamma_{2}+\gamma_{2}\right)+\alpha_{2}\right] \Delta t R^{(m+1) \Delta t}-1
\end{aligned}
$$

Therefore

$$
\begin{aligned}
h_{\Delta}^{\prime}(R) & =\left[\left(2 K_{2}+2 \lambda^{2}\left(\gamma_{1}^{2}+\gamma_{1} \gamma_{2}\right)+\frac{1}{2} K_{1}\right)(\Delta t)^{2}+\left(-2 \alpha_{1}+\alpha_{2}+\beta_{1}^{2}\right.\right. \\
& \left.\left.+\beta_{1} \beta_{2}+\lambda\left(\gamma_{1}^{2}+2 \gamma_{1}+\gamma_{1} \gamma_{2}+\gamma_{2}\right)\right) \Delta t+1\right] \Delta t R^{\Delta t-1} \\
& +(m+1)\left[2 K_{2} \Delta t+2 \lambda^{2} \Delta t\left(\gamma_{2}^{2}+\gamma_{1} \gamma_{2}\right)+\alpha_{2}+\beta_{2}^{2}+\beta_{1} \beta_{2}\right. \\
& \left.+\lambda\left(\gamma_{2}^{2}+\gamma_{1} \gamma_{2}+\gamma_{2}\right)+\frac{1}{2} K_{1} \Delta t\right](\Delta t)^{2} R^{(m+1) \Delta t-1}
\end{aligned}
$$

Choosing $\Delta t_{1}>0$ such that for any $\Delta t<\Delta t_{1}$,

$$
\begin{aligned}
& \left(2 K_{2}+2 \lambda^{2}\left(\gamma_{1}^{2}+\gamma_{1} \gamma_{2}\right)+\frac{1}{2} K_{1}\right)(\Delta t)^{2} \\
+ & \left(-2 \alpha_{1}+\alpha_{2}+\beta_{1}^{2}+\beta_{1} \beta_{2}+\lambda\left(\gamma_{1}^{2}+2 \gamma_{1}+\gamma_{1} \gamma_{2}+\gamma_{2}\right)\right) \Delta t+1>0
\end{aligned}
$$

then for any $R \geq 1, h_{\Delta}^{\prime}(R)>0$.

Moreover for any $\Delta t<\Delta t_{2}:=\frac{2 \alpha_{1}-2 \alpha_{2}-\left(\beta_{1}+\beta_{2}\right)^{2}-\lambda\left(\gamma_{1}+\gamma_{2}\right)^{2}-2 \lambda\left(\gamma_{1}+\gamma_{2}\right)}{4 K_{2}+2 \lambda^{2}\left(\gamma_{1}+\gamma_{2}\right)^{2}+K_{1}}$,

$$
h_{\Delta}(1)=\left[\left(4 K_{2}+2 \lambda^{2}\left(\gamma_{1}+\gamma_{2}\right)^{2}+K_{1}\right) \Delta t\right.
$$




$$
\left.+\left(\beta_{1}+\beta_{2}\right)^{2}+\lambda\left(\gamma_{1}+\gamma_{2}\right)^{2}+2 \lambda\left(\gamma_{1}+\gamma_{2}\right)+2\left(-\alpha_{1}+\alpha_{2}\right)\right] \Delta t<0
$$

Hence, for any $\Delta t<\Delta t_{1} \wedge \Delta t_{2}$, there exists a unique positive constant $R^{*}>1$ such that $h_{\Delta}\left(R^{*}\right)=0$. Therefore we have

$$
\begin{aligned}
R^{k \Delta t}\left|y_{k}\right|^{2}+\sigma \sum_{j=k-m}^{k-1} R^{(j+m+1) \Delta t}\left|y_{j}\right|^{2} & \leq\left|y_{0}\right|^{2}+\sigma \sum_{j=-m}^{-1} R^{(j+m+1) \Delta t}\left|y_{j}\right|^{2} \\
& +M_{k}\left(R^{*}\right):=Z_{k}
\end{aligned}
$$

Noting that the initial data $y_{k}=\psi(k \Delta t)<\infty$ for $k=-m,-m+1, \ldots, 0$ and $M_{k}\left(R^{*}\right)$ is a martingale. Then by Lemma 3 , for $R=R^{*}$ we can conclude that

$$
\lim _{k \rightarrow \infty} Z_{k}<\infty \quad \text { a.s. }
$$

Then by this conclusion we can get from (40), the following relation

$$
\begin{aligned}
\limsup _{k \rightarrow \infty} R^{* k \Delta t}\left|y_{k}\right|^{2} & \leq \limsup _{k \rightarrow \infty}\left[R^{* k \Delta t}\left|y_{k}\right|^{2}+\sigma \sum_{j=k-m}^{k-1} R^{*(j+m+1) \Delta t}\left|y_{j}\right|^{2}\right] \\
& \leq \lim _{k \rightarrow \infty} Z_{k}<\infty \quad \text { a.s. }
\end{aligned}
$$

Noting that $\Delta t=\frac{\tau}{m}$, by (38), we have

$$
\begin{aligned}
& \frac{1-R^{*-\Delta t}}{\Delta t}+2 K_{2} \Delta t+2 \lambda^{2} \Delta t\left(\gamma_{1}^{2}+\gamma_{1} \gamma_{2}\right)+\beta_{1}^{2}+\beta_{1} \beta_{2}+\frac{1}{2} K_{1} \Delta t \\
& \quad+\lambda\left(\gamma_{1}^{2}+2 \gamma_{1}+\gamma_{1} \gamma_{2}+\gamma_{2}\right)-2 \alpha_{1}+\alpha_{2}+\left(2 K_{2} \Delta t+2 \lambda^{2} \Delta t\left(\gamma_{2}^{2}+\gamma_{1} \gamma_{2}\right)\right. \\
& \left.+\beta_{2}^{2}+\beta_{1} \beta_{2}+\frac{1}{2} K_{1} \Delta t+\lambda\left(\gamma_{2}^{2}+\gamma_{1} \gamma_{2}+\gamma_{2}\right)+\alpha_{2}\right) R^{* \tau}=0 .
\end{aligned}
$$

Choose the constant $\Lambda$ such that $R=e^{\Lambda}$.

Define

$$
\begin{aligned}
& h_{\Delta}(\Lambda)=\frac{1-e^{-\Lambda \Delta t}}{\Delta t}+2 K_{2} \Delta t+2 \lambda^{2} \Delta t\left(\gamma_{1}^{2}+\gamma_{1} \gamma_{2}\right)+\beta_{1}^{2}+\beta_{1} \beta_{2}+\frac{1}{2} K_{1} \Delta t \\
& +\lambda\left(\gamma_{1}^{2}+2 \gamma_{1}+\gamma_{1} \gamma_{2}+\gamma_{2}\right)-2 \alpha_{1}+\alpha_{2}+\left(2 K_{2} \Delta t+2 \lambda^{2} \Delta t\left(\gamma_{2}^{2}+\gamma_{1} \gamma_{2}\right)\right. \\
& \left.+\beta_{2}^{2}+\beta_{1} \beta_{2}+\frac{1}{2} K_{1} \Delta t+\lambda\left(\gamma_{2}^{2}+\gamma_{1} \gamma_{2}+\gamma_{2}\right)+\alpha_{2}\right) e^{\Lambda \tau} .
\end{aligned}
$$


Let $\bar{\Lambda}_{\Delta t}=\log \left(R^{*}\right)$, by (42), for any $\Delta t<\Delta t_{1} \wedge \Delta t_{2}$, we have

$$
\begin{aligned}
& h_{\Delta}\left(\bar{\Lambda}_{\Delta t}\right)=\frac{1-e^{-\bar{\Lambda}_{\Delta t} \Delta t}}{\Delta t}+2 K_{2} \Delta t+2 \lambda^{2} \Delta t\left(\gamma_{1}^{2}+\gamma_{1} \gamma_{2}\right)+\beta_{1}^{2}+\beta_{1} \beta_{2}+\frac{1}{2} K_{1} \Delta t \\
& \quad+\lambda\left(\gamma_{1}^{2}+2 \gamma_{1}+\gamma_{1} \gamma_{2}+\gamma_{2}\right)-2 \alpha_{1}+\alpha_{2}+\left(2 K_{2} \Delta t+2 \lambda^{2} \Delta t\left(\gamma_{2}^{2}+\gamma_{1} \gamma_{2}\right)\right. \\
& \left.+\beta_{2}^{2}+\beta_{1} \beta_{2}+\frac{1}{2} K_{1} \Delta t+\lambda\left(\gamma_{2}^{2}+\gamma_{1} \gamma_{2}+\gamma_{2}\right)+\alpha_{2}\right) e^{\bar{\Lambda}_{\Delta t} \tau}=0
\end{aligned}
$$

Noting that $\lim _{\Delta t \rightarrow 0} \frac{1-e^{-\Lambda \Delta t}}{\Delta t}=\Lambda$, then we have

$$
\begin{aligned}
& \lim _{\Delta t \rightarrow 0} h_{\Delta}(\Lambda)=\Lambda+\left(\beta_{1}^{2}+\beta_{1} \beta_{2}+\lambda\left(\gamma_{1}^{2}+2 \gamma_{1}+\gamma_{1} \gamma_{2}+\gamma_{2}\right)-2 \alpha_{1}+\alpha_{2}\right) \\
& \quad+\left(\beta_{2}^{2}+\beta_{1} \beta_{2}+\lambda\left(\gamma_{2}^{2}+\gamma_{1} \gamma_{2}+\gamma_{2}\right)+\alpha_{2}\right) e^{\Lambda \tau}=0
\end{aligned}
$$

By the definition of $\bar{\eta}>0$ in (21), we have that

$$
\lim _{\Delta t \rightarrow 0} \bar{\Lambda}_{\Delta t}=\bar{\eta}
$$

which implies that for any positive constant $\varepsilon \in\left(0, \frac{\bar{\eta}}{2}\right)$, there exists a $\Delta t_{3}>0$ such that for any $\Delta t<\Delta t_{3}$, we have $\bar{\Lambda}_{\Delta t}>\bar{\eta}-2 \varepsilon$.

By using (41) and the definition of $\bar{\Lambda}_{\Delta t}$, it yields

$$
\limsup _{k \rightarrow \infty} e^{\bar{\Lambda}_{\Delta t} k \Delta t}\left|y_{k}\right|^{2}<\infty \quad \text { a.s. }
$$

Letting $\Delta t^{*}=\Delta t_{1} \wedge \Delta t_{2} \wedge \Delta t_{3}$, therefore for any $\Delta t<\Delta t^{*}$, we obtain

$$
\limsup _{k \rightarrow \infty} \frac{1}{k \Delta t} \log \left|y_{k}\right| \leq-\frac{\bar{\eta}}{2}+\varepsilon \quad \text { a.s. }
$$

which gives (20). This completes the proof of the Theorem 6 .

\section{Numerical experiments}

In this section, we introduce a model of SDDE with jump process whose the coefficients are nonlinear and its simulation to illustrate the almost sure exponential stability of the Milstein method. 
Consider the following scalar nonlinear SDDE with jumps

$d x(t)=(-4 x(t)+2 x(t-1)) d t+x(t) \cos (x(t-1)) d W(t)+\frac{x\left(t^{-}\right)}{1+x^{2}\left(t^{-}\right)+y^{2}\left(t^{-}\right)} d N(t), t>0$,

with the initial data $x(t)=\psi(t)=2^{4}$ for $t \in[-1,0]$, where $W(t)$ is a scalar Brownian motion and $N(t)$ is a scalar Poisson process with parameter $\lambda=1$. We can deduce that the drift and diffusion coefficients, as well the functions $h$ and $L^{1} g$ satisfy the conditions (8)-(12), (17) and (19) with $\alpha_{1}=4, \alpha_{2}=2, \beta_{1}=$ $1, \beta_{2}=0, \gamma_{1}=1$ and $\gamma_{2}=0$. According to Theorem 4, the solution to Equation (47) is almost surely exponentially stable.

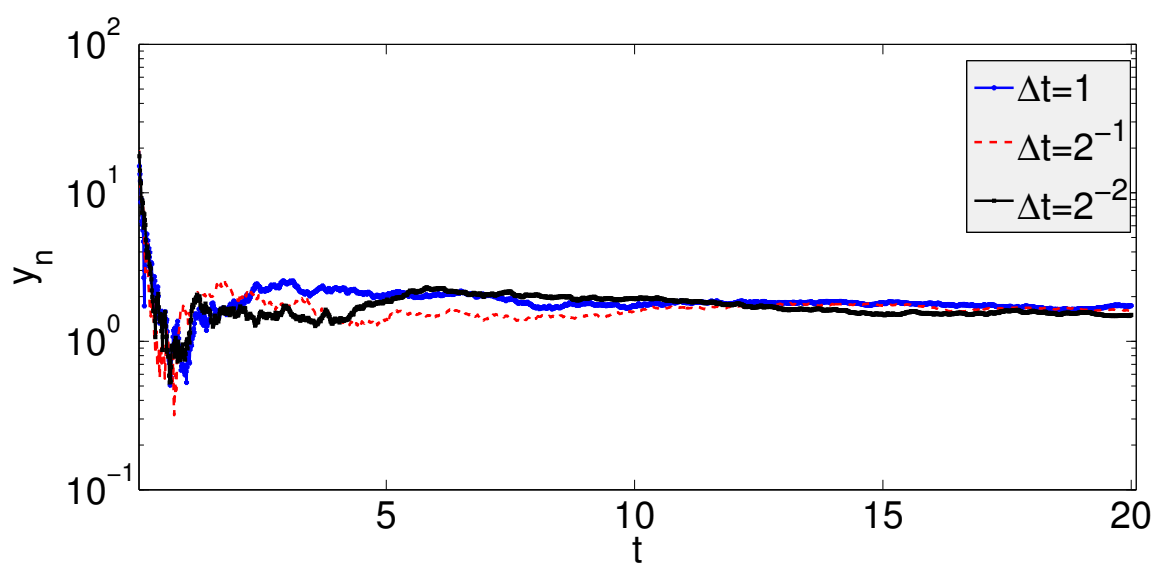

Figure 1: Almost sure exponential stability of $y_{n}$ with initial data $x(t)=2^{4}$.

Choosing the stepsizes $\Delta t=1,2^{-1}, 2^{-2}$ and taking the average of $10^{3}$ sample paths, Theorem 6 shows that the Milstein method for equation (47) is almost surely exponentially stable for any $\Delta t<\Delta t^{*}$, which is shown in Figure 1.

\section{Conclusion}

In this paper, we consider the almost sure exponential stability of the Milstein method for the SDDEs with Poisson jumps. For this purpose, we derive some sufficient conditions for the drift, diffusion and Poisson coefficients. Also, the semimartingale theorem of Doob is given, and using martingale property of the compensated Poisson process, we reach the almost sure exponential stability. 
Finally, we present an example simulated and the numerical experiments verify the results obtained from theory.

\section{References}

[1] C. T. H. Baker and E. Buckwar, Numerical analysis of explicit one-step methods for stochastic delay differential equations, LMS Journal of Computation and Mathematics. 3 (2000) pp. 315-335.

[2] R. Cont and P. Tankov, Financial Modelling with Jump Processes, Financial Mathematics Series. Chapman and Hall/CRC Press, London. 2003.

[3] C. T. H. Baker and E. Buckwar, Exponential stability in p-th mean of solutions and of convergent Euler-type solutions of stochastic delay differential equations, Journal of Computational and Applied Mathematics. 184 (2005), pp. 404-427.

[4] K. Sobczyk, Stochastic Differential Equations: with Application to Physics and Engineering, Kluwer Academic, Dordrecht. 1991.

[5] J. Tan and H. Wang, Mean-square stability of the Euler-Maruyama method for stochastic differential delay equations with jumps, International Journal of Computer Mathematics. 88 (2011), pp. 421-429.

[6] A. Rodkina and H. Schurz, Almost sure asymptotic stability of drift-implicit $\theta$-methods for bilinear ordinary stochastic differential equations in $\mathbb{R}^{1}$, Journal of Computational and Applied Mathematics. 180 (2005), pp. 13-31.

[7] N. Liberati and E. Platen, Approximation of jump diffusions in finance and economics, Comput. Econom. 29 (2007), pp. 283-312.

[8] W. Zhang, J. Ye and H. Li, Stability with general decay rates of stochastic differential delay equations with Poisson jumps and Markovian switching, Statistics and Probability Letters. 92 (2014), pp. 1-11.

[9] X. Mao, C. Yuan and J. Zouc, Stochastic differential delay equations of population dynamics, Journal of Mathematical Analysis and Applications. 304 (2005), pp. 296-320.

[10] P. E. Kloeden and E. Platen, Numerical Solution of Stochastic Differential Equations, Applications of Mathematics(New York), 23, Springer-Verlag, Berlin. 1992.

[11] L. Wang, C. Mei and H. Xue, The semi-implicit Euler method for stochastic differential delay equation with jumps, Applied Mathematics and Computation. 192 (2007), pp. $567-578$.

[12] C. Qian, Global attractivity in a delay differential equation with application in a commodity model, Applied Mathematics Letters. 24 (2011), pp. 116-121.

[13] Q. Li and S. Gan, Almost sure exponential stability of numerical solutions for stochastic delay differential equations with jumps, Journal of Applied Mathematics and Computing. 37 (2011), pp. 541-557.

[14] X. Mao, Stochastic Differential Equations and their Applications, Horwood. Chichester, 1997.

[15] X. Mao, LaSalle-type theorems for stochastic differential delay equations, Journal of Mathematical Analysis and Applications. 236 (1999), pp. 350-369. 
[16] X. Mao, A note on the LaSalle-type theorems for stochastic differential delay equations, Journal of Mathematical Analysis and Applications. 268 (2002), 125-142.

[17] X. Mao and S. Sabanis, Numerical solutions of stochastic differential delay equations under local lipschitz condition, Journal of Computational and Applied Mathematics. 151 (2003), pp. 215-227.

[18] X. Mao, F. Wu and P. E. Kloeden, Discrete razumikhin-type technique and stability of the Euler maruyama method to stochastic functional differential equations, Discrete and Continuous Dynamical Systems. 33 (2013), pp. 885-903.

[19] Q. Li, S. Gan and X. Wang, Compensated stochastic theta methods for stochastic differential delay equations with jumps, International Journal of Computer Mathematics. $\mathbf{5}$ (2013), pp. 1057-1071.

[20] G. N. Milstein and M. V. Tretyakov, Stochastic Numerics for Mathematical Physics, Scientific Computation, Springer-Verlag, Berlin. 2004.

[21] X. Qu and C. Huang, Delay-dependent exponential stability of the backward Euler method for nonlinear stochastic delay differential equations, International Journal of Computer Mathematics. 89 (2012), pp. 1039-1050.

[22] A. Rathinasamy and K. Balachandran, Mean-square stability of Milstein method for linear hybrid stochastic delay integro-differential equations, Nonlinear Analysis: Hybrid Systems. 2 (2008), pp. 1256-1263.

[23] J. Shi, Optimal control for stochastic differential delay equations with poisson jumps and applications, Journal of Computational and Applied Mathematics. (2015), doi:10.1515/rose-2014-0028.

[24] D. Wang, C. Huanga and S. Gan, Delay-dependent stability analysis of numerical methods for stochastic delay differential equations, Journal of Computational and Applied Mathematics. 236 (2012), pp. 3514-3527.

[25] Z. Wang and C. Zhang, An analysis of stability of Milstein method for stochastic differential equations with delay, Computers and Mathematics with Applications. 51 (2006), pp. $1445-1452$.

[26] F. Wu, X. Mao and L. Szpruch, Almost sure exponential stability of numerical solutions for stochastic delay differential equations, Numerische Mathematik. 115 (2010), pp. 681-697.

[27] R. C. Merton, Option pricing when underlying stock returns are discontinuous, Journal of Financial Economics. 3 (1976), pp. 125-144.

[28] R. S. Liptser and A. N. Shiryaev, Theory of Martingale, Kluwer Academic Publishers. Dordrecht, 1989.

[29] A. N. Shiryaev, Probability, Springer. Berlin, 1996. 